\title{
Urinary metabolites of type 2 diabetes rats fed with palm oil-enriched high fat diet
}

\author{
Xuan-Yi Sim ${ }^{\text {a,b }}$, Baharudin Ibrahim ${ }^{\text {c,**}, ~ L a y-H a r n ~ G a m ~}{ }^{\text {a,b,* }}$ \\ ${ }^{a}$ USM-RIKEN International Centre of Aging Science, USM, 11800 Minden, Penang, Malaysia \\ ${ }^{\mathrm{b}}$ School of Pharmaceutical Sciences, Universiti Sains Malaysia, 11800 Minden, Penang, Malaysia \\ ${ }^{\mathrm{c}}$ Faculty of Pharmacy, Universiti of Malaya, Kuala Lumpur, 50603, Malaysia
}

\section{A R T I C L E I N F O}

\section{Keywords:}

Metabolites

NMR

Diabetes

High fat diet

Metformin

\begin{abstract}
A B S T R A C T
High fat diet (HFD) is one of the risk factors of obesity and diabetes. Recommended diet regimen for diabetes is difficult to abide by especially for HFD as it adds flavour to the taste buds. In this study, palm oil-enriched HFD and normal diet were fed to nicotinamide-induced type 2 diabetes rats, respectively for six weeks. Additionally, metformin, a common drug used to treat diabetes was given to rats under treatment groups. We evaluated the change of urinary metabolites of diabetes rats fed with palm oil-enriched HFD, and also after metformin treatment. Rats were divided into six-groups with different feeding diets, disease condition and with or without metformin treatment. Rats' urine were collected at the end of six weeks feeding program and subjected to $1 \mathrm{H}-$ NMR and multivariate data analysis to evaluate their metabolite profiles. At the early phase of diabetes, metabolites changes in diabetic rats were associated with the disease itself. Our data showed that continuous consumption of HFD altered various metabolic pathways of diabetic rats and caused detrimental effects to the rats. On the other hand, metformin treatment combined with normal diet lessened the physiological impacts caused by diabetes condition.
\end{abstract}

\section{Introduction}

Diabetes is one of the most common non-communicable diseases affecting $9.3 \%$ of world population, it is characterised by high blood glucose levels [1]. Type 2 diabetes mellitus (T2DM) is the most common type of diabetes. Obesity is strongly associated with T2DM as it is one of the leading causes of insulin resistance, a typical characteristic of T2DM [2]. The most common cause of obesity is due to high fat diet (HFD) consumption. HFD is defined as the dietary fat intake $>30 \%$ of the total diet [3] and it has been linked to metabolic diseases, including obesity, diabetes, and cardiovascular diseases.

Palm oil is the most consumed fat in Asia, Africa, and Europe [4, 5]. Palm oil is a very popular household cooking oil and ingredient in food industry due to its texture, fragrance, and neutral taste compared to other vegetable oils. Besides, palm oil has a high smoke point at $230^{\circ} \mathrm{C}$, making it suitable for most cooking methods [5]. Palm oil has a balanced ratio of unsaturated and saturated fatty acids. Palmitic acid is the major saturated fatty acid in palm oil (45\%) and followed by stearic acid (5\%). Additionally, palm oil also contains $40 \%$ oleic acid (monounsaturated fatty acid) and 10\% linoleic acid (polyunsaturated fatty acid) [4]. Palmitic acid (16:0), a long chain saturated fatty acid is the most abundant saturated fatty acid present naturally in vegetable oil, animal fat, and human milk fat [6]. Among the fatty acids, long-chain saturated fatty acid, such as palmitic acid, was said to be lipotoxic compared to medium-chain (C6-C12) saturated fatty acids or unsaturated fatty acids [7, 9]. Long-chain saturated fatty acid was reported to promote insulin resistance, inflammation, and fat storage [7, 8, 9]. Nevertheless, a recent study has shown that long chain saturated fatty acids were well tolerated by human $\beta$-cells and therefore may not cause insulin resistance [9].

Besides dietary fat, lifestyle modifications such as healthy eating and physical activity, are essential for diabetes management [10]. When lifestyle interventions fail or it becomes hard to manage the blood glucose levels, medical treatment becomes an important option. Metformin (dimethylbiguanide) is one of the most common drugs, and the first-line treatment for T2DM [11]. Metformin is an antihyperglycemic agent that decreases hepatic glucose production, increases peripheral glucose uptake, and halts gastrointestinal glucose absorption [12].

\footnotetext{
* Corresponding author.

$* *$ Corresponding author.

E-mail addresses: baharudin.ibrahim@um.edu.my (B. Ibrahim), layharn@usm.my (L.-H. Gam).
} 
In this study, NMR-based metabolomics approach was applied to evaluate the urinary metabolites. This technique consists of a combination of 1H-NMR spectroscopic profiling and multivariate statistical analysis. It is a powerful tool for identifying changes in metabolites profiles of biological samples. It is a common approach used in diet and drug intervention for diseases [13]. Urine has high medical diagnostic and biomarker values as it does not involve body homeostasis; it can be collected easily and non-invasively $[14,15]$. Biomarkers related to diet, or food intervention have been discovered via urinary metabolomics-based analysis. For instance, proline betaine and hydroxyproline betaine are biomarkers of citrus juice consumption, while creatinine, carnitine, acetylcarnitine and trimethylamine-N-oxide are the biomarkers of red meat consumption. On the other hand, formate, citrulline, taurine and isocitrate are used as markers for sugar sweetened beverages [16, 17, 18, 19]. Furthermore, urine metabolomics is useful to detect endogenous metabolic variation and kidney function efficiency. This is an added value to our study because the kidneys are commonly affected by diabetes.

Although studies on the metabolites profile of diabetic rats [20, 21, $22,23,24]$ had been reported, where the pathogenesis and progression of the disease were evaluated by elucidation of urinary metabolites profile. Nevertheless, study on urinary metabolites changes due to palm oil-enriched HFD on diabetic rats is new to our knowledge. We think this information is important because diabetes cases are on the rise as palm oil is heavily used in cooking preparations, especially frying, which adds taste and texture to the palate [25]. Not to mention, these food can hardly be resisted by diabetes patients. Therefore, we aimed to evaluate the impact of palm oil-enriched HFD upon T2DM rat models' urinary metabolite profiles using $1 \mathrm{H}-\mathrm{NMR}$ and multivariates data analysis, which was hoped to provide a deeper understanding on the impact of palm oil-enriched HFD to the existing metabolic pathways of the disease.

\section{Materials and methods}

\subsection{Animal study}

Male Sprague Dawley rats weighing $300 \pm 50 \mathrm{~g}$ were obtained from Animal Research Center and Service, Universiti Sains Malaysia. The rats were housed individually in plastic cages with $12 \mathrm{~h}$ of light/dark cycles and were acclimatised for a week before the study started. All experiment procedures were approved by the Animal Ethics Committee Universiti Sains Malaysia (USM/Animal Ethics Approval/2016/(717)).

\subsection{Induction of diabetes mellitus}

The rats were induced to diabetes by nicotinamide (Sigma, USA) and streptozotocin (Sigma, USA) according to Akbarzadeh et al (22). Nicotinamide dissolved in saline (110 $\mathrm{mg} / \mathrm{kg}$ of rat body weight) was administered to the rats through intraperitoneal injection. After $15 \mathrm{~min}$, streptozotocin $(65 \mathrm{mg} / \mathrm{kg}$ of rat body weight), which was dissolved freshly in $0.1 \mathrm{M}$ sodium citrate dihydrate (Sigma, USA), pH 4.5 was injected intraperitoneally to the rats. Control rats were given only the vehicle solution (saline and citrate buffer). The rats were monitored for four weeks before separated into different groups. Rats with fasting blood glucose levels $>7 \mathrm{mmol} / \mathrm{L}$ were classified as diabetic rats. The experimental groups are shown in Table 1.

\subsection{Diet/feed}

The normal diet (ND) and HFD were self-prepared by mixing distilled water with the commercial feed powder (contained $12 \%$ of fat content) (Altromin, Germany) at 1:1 ratio and baked in an oven overnight at 40 ${ }^{\circ} \mathrm{C}$. HFD was prepared by adding $22.4 \%$ (v/w) palm olein cooking oil (Buruh Nam Loong, Malaysia), making a total of $39 \%$ fat content. The final feed products were sent for further analysis and the final composition of the feeds was obtained. Metformin $\mathrm{HCl}$ Tablet $500 \mathrm{mg}$
Table 1. The experimental group for the study.

\begin{tabular}{llllr}
\hline Group & Non-induced/Induced rat & Diet & Metformin & $\mathrm{n}$ \\
\hline NN & Control & ND & No & 6 \\
NH & Control & HFD & No & 6 \\
DN & Diabetic & ND & No & 6 \\
DNM & Diabetic & ND & Yes & 6 \\
DH & Diabetic & HFD & No & 6 \\
DHM & Diabetic & HFD & Yes & 6 \\
\hline
\end{tabular}

NN (control rat fed with ND); NH (control rat fed with HFD); DN (diabetic rat fed with ND); DNM (Diabetic rat fed with ND treated with metformin); DH (diabetic rat fed with HFD); DHM (diabetic rat fed with HFD treated with metformin).

(Dynapharm, Malaysia) was crushed and mixed with $1 \%$ carboxymethylcellulose (R\&M Chemicals). The oral gavage was given twice daily. Vehicle ( $1 \%$ carboxymethylcellulose) was given to the rats that were not given metformin. The feeding programme was 6 weeks. Water (ad libitum) was provided throughout the study.

\subsection{Fasting blood glucose}

Fasting blood glucose was measured for every rats in the groups ( $\mathrm{n}=$ 6). The measurement was first taken a week before nicotinamide/ streptozotocin induction (P1) and then weekly from the starting point of feeding program until the sixth week of feeding program (W0 to W6) by using Glucometer (Accu-chek Performa, Roche). A drop of blood was taken from the rat tail. The rats were fasted for $12 \mathrm{~h}$ before measurement was taken.

\subsection{Urine collection}

At the end of the feeding period ( 6 weeks), urine was collected by placing the rat in a metabolic cage for $24 \mathrm{~h}$. A volume of $500 \mu \mathrm{l}$ of $1 \%$ sodium azide (Sigma, USA) was added to the urine collection bottles. The urine was stored at $-80^{\circ} \mathrm{C}$ until further analysis.

\subsection{Sample preparation for $1 H$-NMR analysis}

The urine samples were thawed. The thawed urine was centrifuged at $8000 \mathrm{rpm}$ at $4{ }^{\circ} \mathrm{C}$ for $5 \mathrm{~min}$. A volume of $400 \mu \mathrm{l}$ of the supernatant was pipetted to a new Eppendorf tube and mixed with $200 \mu \mathrm{l}$ of phosphate buffer. The phosphate buffer contained $28.85 \mathrm{~g}$ disodium hydrogen phosphate, $\mathrm{Na}_{2} \mathrm{HPO}_{4}$ (R\&M Chemicals, UK), $5.25 \mathrm{~g}$ sodium dihydrogen phosphate, $\mathrm{NaH}_{2} \mathrm{PO}_{4}$ (R\&M Chemicals, UK), $1 \mathrm{mM}(0.172 \mathrm{~g}$ ) sodium 3(trimethylsilyl)-propionate-2,2,3,3-d4, TSP (ARMAR AG, Switzerland) and $200 \mathrm{ml}$ of deuterium oxide, $\mathrm{D}_{2} \mathrm{O}$ (ARMAR AG, Switzerland). The volume was topped up to $1 \mathrm{~L}$ by distilled water. Urine and phosphate buffer mixture were vortexed for a few seconds. Finally, $550 \mu \mathrm{l}$ of the mixture was transferred to a $5 \mathrm{~mm}$ NMR tube and capped.

\subsection{H-NMR analysis}

1H-NMR spectra of urine were collected without magic-angle spinning at $300 \mathrm{~K} \pm 0.1$ on a Bruker Advance III $500 \mathrm{MHz}$ (Bruker, Fallanden, Switzerland) spectrometer with a room temperature $\left(25{ }^{\circ} \mathrm{C}\right)$ observe detection probe equipped with z-gradients and an automatic sample changer. The temperature was calibrated using $99.8 \%$ deuterated methanol. Each sample was held for $5 \mathrm{~min}(300 \mathrm{~s})$ in the magnet for thermal equilibration before measurement. The probe was tuned and matched automatically and the magnetic field was locked to the solvent (urine $+\mathrm{D}_{2} \mathrm{O}$ ), optimised for urine and shimmed using an automatic routine. Automatic $1 \mathrm{H}$ pulsecal calibration (pulsecal) was performed on each sample to avoid the effect of sample variation. Each sample was subjected to 32 scans with 4 dummy scans, $0.16 \mathrm{~Hz} /$ point and relaxation 
delay (D1) of $4 \mathrm{~s}$. Water suppression was achieved to suppress water signal at 4.74-4.90 ppm through weak radio frequency radiation during recycle delay. Water residual was further cleaned up by pulse field gradient. The spectra were recorded and processed using TopSpin 3.2 (Bruker Fallanden, Switzerland). The spectra were processed according to pre-set automated routine where the spectra were phased, baseline corrected and calibrated to TSP at $0.0 \mathrm{ppm}$. Automatic zero-order phase correction was applied while first-order phase correction was optimised and adjusted according to Bruker standard during setup. All samples followed the same acquisition and processing parameters. The spectrum's quality was attained by checking the line width at half height of TSP peak. It was considered satisfactory if the value was $<2 \mathrm{~Hz}$.

\subsection{Multivariate data analysis and metabolites identification}

NMR data spectrum was reduced and segmented into 250 bins of 0.04 ppm width from the region of 0.02-10 ppm using AMIX software (Bruker Analytische Messtechnik, Rheinstetten, Germany). NMR data were normalised to total peak intensity and scaled to TSP (reference region). The Amix file was exported to Microsoft Excel for SIMCA analysis to generate OPLS-DA. Water (4.74-4.90 ppm) and urea regions (5.70-5.90 ppm) were excluded from the analysis to avoid effect of inter-sample variation due to water suppression. Glucose regions (3.22-3.98 ppm, 4.62-4.66 ppm, and 5.18-5.30 ppm) of the diabetic rats was excluded. For the comparisons that involved metformin treated groups, metformin region (3.038-3.055 ppm) was excluded in OPLS-DA analysis. The data were scaled using auto Pareto scaling in SIMCA-P 13 (Umetrics, Umea, Sweden) and OPLS-DA analysis, from which a VIP plot was generated to identify the important regions that contributed to the difference between the groups. Only the NMR peaks in regions with VIP score $>1$ were integrated, and subsequently a second OPLS-DA analysis was carried out using the integrated data. Identification of the metabolites with VIP score $>1$ was carried out by matching of their respective chemical shift, multiplicity (singlet, doublet or quartet) and relative intensity values with the values of known metabolites in the databases from Biological Magnetic Resonance Bank (BMRB), Human Metabolome Database (HMDB) and Chenomx profiler. A match indicates the identity of the metabolite.

\section{Results}

\subsection{The effect of HFD and metformin on fasting blood glucose}

In this study, all the rats were with normal fasting blood glucose (FBG) $<5.5 \mathrm{mmol} / \mathrm{L}$ before the induction of diabetes by nicotinamide and streptozotocin. Figure 1 shows the chart for the blood glucose of rats $(n=6)$ in each groups (mean $\pm S D$ ). The parameter to define diabetes condition was FBG $>7 \mathrm{mmol} / \mathrm{L}[26,27]$. A drastic increase in FBG (p > 0.05 ) within the first week of HFD feeding program was observed in diabetic rats, while for the ND fed diabetes rats, a gradual increase in FBG was observed. The FBG for diabetic rats reached a plateau at 19.4-22.9 $\mathrm{mmol} / \mathrm{L}$ throughout the remaining feeding period (up to week-6). Metformin treatment reduced FBG of diabetic rats to a level ranged between (11.0-16.4 mmol/L). HFD did not alter the FBG of control rats throughout the feeding program.

\subsection{The effect of HFD on urinary metabolites of control and diabetic rats}

Table 2 shows the comparison between groups of animals that was carried out in this study. The effect of HFD on the rats was carried out by comparing 1) between control rat fed with ND (NN) and control rat fed with HFD (NH); 2) between diabetic rat fed with ND (DN) and diabetes rat with HFD (DH); 3) between diabetic rat fed with ND treated with metformin (DNM) and diabetic rat fed with HFD treated with metformin (DHM). R2Y is the fraction of variance of Y matric while Q2Y is the predictive accuracy of the model, both these values are used in OPLS_DA analysis. OPLS-DA is a useful statistical analysis tool to generate useable information from a challenging and non-systematic data, when a

Table 2. Group comparison: the sensitivity, specificity, accuracy, R2Y, and Q2Y of OPLDS-DA models.

\begin{tabular}{llllll}
\hline Group comparison & Sensitivity,\% & Specificity,\% & Accuracy,\% & R2Y & Q2Y \\
\hline High Fat Diet & & & & & \\
NN versus NH & 100 & 100 & 100 & 0.832 & 0.455 \\
DN versus DH & 83.33 & 100 & 91.67 & 0.760 & 0.401 \\
DNM versus DHM & 85.71 & 100 & 92.31 & 0.700 & 0.483 \\
Diabetes & & & & & \\
NN versus DN & 80 & 100 & 90.91 & 0.86 & 0.747 \\
NH versus DH & 100 & 100 & 100 & 0.97 & 0.397 \\
Metformin & & & & & \\
DN versus DNM & 100 & 83.33 & 91.67 & 0.967 & 0.677 \\
DH versus DHM & 100 & 71.43 & 86.67 & 0.575 & 0.42 \\
\hline
\end{tabular}

NN (control rat fed with ND); NH (control rat fed with HFD); DN (diabetic rat fed with ND); DNM (Diabetic rat fed with ND treated with metformin); DH (diabetic rat fed with HFD); DHM (diabetic rat fed with HFD treated with metformin). R2Y (the fraction of variance of Y matric); Q2Y (the predictive accuracy of the model).

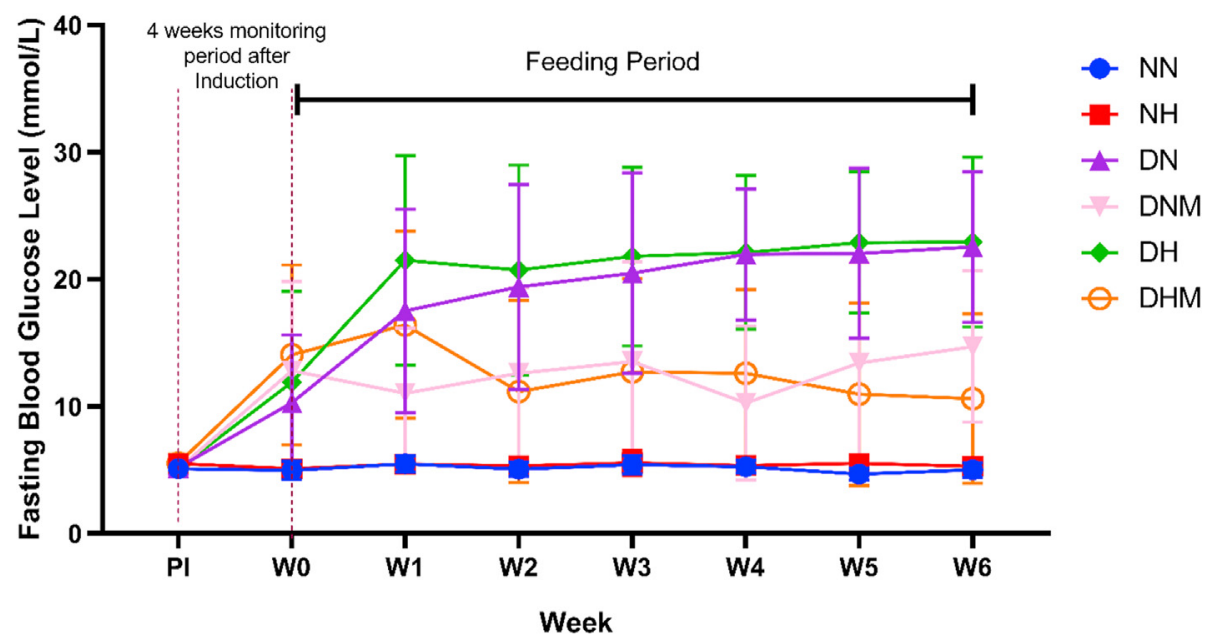

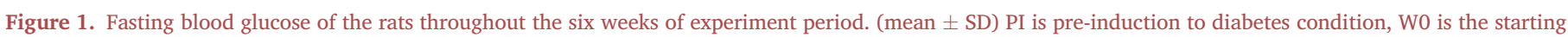

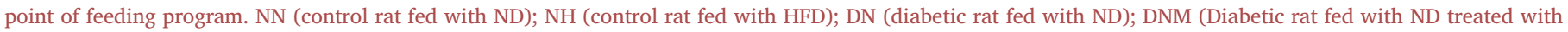
metformin); DH (diabetic rat fed with HFD); DHM (diabetic rat fed with HFD treated with metformin). 
separation was generated between data contributors from two comparison groups, this indicated that the two groups are of significant different statistically based on the data contributors [28]. The OPLS-DA showed clear separation for all three comparisons (Figure 2A-C). All the comparisons had sensitivity, specificity, and accuracy above $83 \%$ (Table 2). The metabolites that contributed to the separation (VIP $>1$ ) were identified and the identities of the compounds are listed in Table 3. The compounds labelled as unknown were the metabolites that cannot be identified using Biological Magnetic Resonance Bank and the other two databases. In the comparison between ND-fed and HFD-fed of control rats, HFD caused the increase of citrate, sarcosine, and phenylacetylglycine while HFD decreased hippurate, trimethylamine-N-oxide (TMAO), 2-oxoglutarate, creatinine, and taurine. As for the comparison between ND-fed and HFD-fed of diabetic rats; acetoacetate, citrate, creatinine, allantoin, acetone, oxoglutarate and 3-hydroxybutyrate were increased by HFD. Lastly, for the comparison between the metformin treated groups of ND-fed and HFD-fed of diabetic rats; citrate, allantoin, methylamine, sarcosine, methylguanidine, and creatinine were increased by HFD while lactate was decreased by HFD.

\subsection{The effect of diabetes on urinary metabolites}

The effect of diabetes on rats was evaluated by comparing between 1 ) control rat fed with ND (NN) and diabetic rat fed with ND (DN); 2) between control rat fed with HFD (NH) and diabetes rat with HFD (DH). Both comparisons showed clear separation in OPLS-DA analysis (Figure 2D and E). The sensitivity, specificity and accuracy level for both comparisons were above $80 \%$ (Table 2). The metabolites that contributed to the separation (VIP $>1$ ) were identified and listed in Table 4. The metabolites that showed reduction in intensity due to diabetes, regardless of the type of diet fed were hippurate, citrate, allantoin, creatinine, succinate, sarcosine and 2-oxoglutarate. Lactate was reduced in diabetic rats fed with ND. On the other hand, cis-aconitate, penylacetylglycine, and acetyglycine were decreased in diabetic rats fed with HFD.

\subsection{The effect of metformin on urinary metabolites of diabetic rats}

The effect of metformin treatment was evaluated by comparing 1) between diabetic rat fed with ND (DN) and diabetic rat fed with ND treated with metformin (DNM); 2) between diabetic rat fed with HFD (DH) and diabetic rat fed with HFD treated with metformin (DHM). The metformin region was excluded in the OPLS-DA analysis as intact metformin was excreted in urine after the treatment [29]. Both comparisons showed clear separation in OPLS-DA analysis (Figure 2F and G). The sensitivity, specificity, and accuracy levels for both comparisons were above $71 \%$ (Table 2). The metabolites that contributed to the separation $(\mathrm{VIP}>1)$ were identified and listed in Table 5 . In the diabetic rats fed with $\mathrm{ND}$, metformin caused the increase of lactate and 3-hydroxybutyrate. On the other hand, lactate, allantoin, citrate, succinate, sarcosine and creatinine were increased by metformin in diabetic rats fed with HFD. Metformin also caused the decrease of 3-hydroxybutyrate in diabetic rats fed with HFD.

\section{Discussion}

Urine has good diagnostic values, because it is not involved in body homeostasis $[14,15]$. Thus, the analysis of urinary metabolites provides useful information on physiological changes of the body. HFD is defined as diet that contained $>30 \%$ fat [3]. According to this definition, many of our daily food intakes are consisting of HFD. Vegetable oil is a preferred fat used in food preparation, this is due to health concern with the perception that animal fat is a bad choice for health [30]. Therefore, this study was designed to understand the physiological impact of palm oil-enriched HFD on health through NMR analysis of urinary metabolites. In addition, metformin was given to diabetes rats and the metabolites profiles were evaluated. Urea, the most abundant metabolite in urine, was excluded from the analysis due to its potential interference with water suppression effect by 1H-NMR [31].

The urinary metabolites profiles have shown that feeding of palm oilenriched HFD caused metabolites changes to diabetes rats. In general, most of these metabolites were the components of known metabolic pathways, although most of these were the catabolism processes, the data may be biased as only monomolecular organic compounds were captured in the analysis.

The altered levels of metabolites detected showed a high possibility that tricarbocylic acid cycle (TCA) was affected. Figure 3 shows TCA cycle, and the metabolites identified in this study with altered levels of excretion in the urine are as labelled.

TCA cycle is the key metabolic pathway involved in various metabolic networks, including carbohydrates, fat, and protein metabolisms. TCA cycle feeds NADH into oxidative phosphorylation pathway to produce energy required by body in the form of ATP [33]. A parallel decreased in
Effect of High Fat Diêt
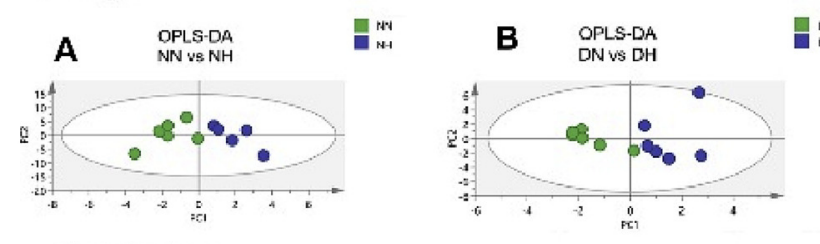

Effect of Diabetes
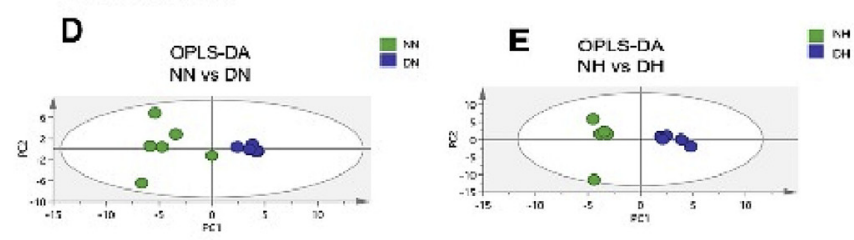

Effect of Metformin

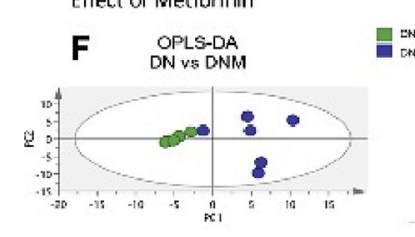

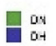

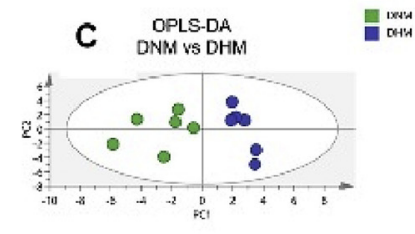

Figure 2. OPLS-DA score scatter plots of all the rat's comparison models. Panel A: OPLS_DA of NN vs NH; Panel B: OPLS-DA of DN vs DH; Panela C: OPLS-DA of DNM vs DHM; Panel D; OPLS-DA of NN vs DN; Panel E: OPLS-DA of NH vs DH; Panel F: OPLS-DA of DN vs DNM; Panel G: OPLS-DA of DH vs DHM. NN (control rat fed with ND); $\mathrm{NH}$ (control rat fed with HFD); DN (diabetic rat fed with ND); DNM (Diabetic rat fed with ND treated with metformin); DH (diabetic rat fed with HFD); DHM (diabetic rat fed with HFD treated with metformin). 
Table 3. Metabolites that were altered by HFD.

\begin{tabular}{|c|c|c|c|}
\hline \multicolumn{4}{|c|}{ ND fed control rats versus HFD fed control rats } \\
\hline Compound & $\begin{array}{l}\text { Chemical shift, } \\
\text { ppm }\end{array}$ & $\begin{array}{l}\text { Relative Intensity } \\
\text { NN }\end{array}$ & $\begin{array}{l}\text { Relative Intensity } \\
\mathrm{NH}\end{array}$ \\
\hline \multirow[t]{2}{*}{ Hippurate } & $7.8226-7.8542(d)$ & $3.531 \pm 1.738$ & $3.265 \pm 1.555$ \\
\hline & $7.538-7.581(t)$ & $3.332 \pm 1.671$ & $3.053 \pm 1.347$ \\
\hline \multirow[t]{2}{*}{ Citrate } & $2.5277-2.5681(d)$ & $4.603 \pm 2.906$ & $5.295 \pm 2.566$ \\
\hline & $2.6689-2.713(d)$ & $2.848 \pm 1.693$ & $3.179 \pm 1.698$ \\
\hline TMAO & $3.264-3.27(\mathrm{~s})$ & $1.209 \pm 0.545$ & $0.686 \pm 0.231$ \\
\hline \multirow[t]{2}{*}{ Sarcosine } & $2.722-2.734(\mathrm{~s})$ & $1.001 \pm 0.395$ & $1.198 \pm 0.497$ \\
\hline & $3.583-3.593(\mathrm{~s})$ & $0.786 \pm 0.289$ & $1.257 \pm 0.596$ \\
\hline 2-oxoglutarate & $2.4306-2.466(t)$ & $1.347 \pm 0.661$ & $1.212 \pm 0.877$ \\
\hline \multirow[t]{2}{*}{ Creatinine } & $3.035-3.052(\mathrm{~s})$ & $9.758 \pm 4.004$ & $6.859 \pm 2.363$ \\
\hline & $4.048-4.057(s)$ & $6.927 \pm 3.017$ & $4.709 \pm 1.656$ \\
\hline \multirow[t]{2}{*}{ Phenylacetylglycine } & $3.676-3.683(\mathrm{~s})$ & $1.027 \pm 0.524$ & $1.515 \pm 0.392$ \\
\hline & $3.75-3.761(d)$ & $1.936 \pm 0.636$ & $2.415 \pm 0.767$ \\
\hline Taurine & $3.4149-3.4453(t)$ & $0.676 \pm 0.317$ & $0.362 \pm 0.109$ \\
\hline Unknown & $3.593-3.601(\mathrm{~s})$ & $1.121 \pm 0.527$ & $0.632 \pm 0.222$ \\
\hline Unknown & $3.642-3.653(s)$ & $0.976 \pm 0.255$ & $1.506 \pm 0.939$ \\
\hline Unknown & $3.654-3.66(s)$ & $0.839 \pm 0.346$ & $1.390 \pm 0.731$ \\
\hline Unknown & $3.669-3.674(s)$ & $0.524 \pm 0.207$ & $0.820 \pm 0.333$ \\
\hline Unknown & $3.701-3.711(\mathrm{~s})$ & $1.250 \pm 0.490$ & $0.798 \pm 0.066$ \\
\hline Unknown & $3.721-3.728(\mathrm{~s})$ & $0.785 \pm 0.255$ & $1.339 \pm 0.526$ \\
\hline Unknown & $3.815-3.827(s)$ & $1.438 \pm 0.483$ & $1.727 \pm 0.697$ \\
\hline \multicolumn{4}{|c|}{ ND fed diabetic rats versus HFD fed diabetic rats } \\
\hline Compound & $\begin{array}{l}\text { Chemical shift, } \\
\text { ppm }\end{array}$ & $\begin{array}{l}\text { Relative Intensity } \\
\text { DN }\end{array}$ & $\begin{array}{l}\text { Relative Intensity } \\
\text { DH }\end{array}$ \\
\hline Acetoacetate & $2.282-2.288(\mathrm{~s})$ & $0.055 \pm 0.026$ & $1.859 \pm 4.198$ \\
\hline \multirow[t]{2}{*}{ Citrate } & $2.519-2.567$ (d) & $1.820 \pm 0.649$ & $3.479 \pm 1.660$ \\
\hline & $2.688-2.715$ (d) & $1.707 \pm 0.639$ & $3.110 \pm 1.346$ \\
\hline \multirow[t]{2}{*}{ Creatinine } & $3.043-3.053(\mathrm{~s})$ & $0.7440 \pm 0,439$ & $1.407 \pm 0.448$ \\
\hline & $4.058-4.065(\mathrm{~s})$ & $0.630 \pm 0.284$ & $1.084 \pm 0.354$ \\
\hline Allantoin & $5.388-5.41(\mathrm{~s})$ & $1.214 \pm 0.404$ & $1.674 \pm 0.411$ \\
\hline Acetone & $2.234-2.244(\mathrm{~s})$ & $0.154 \pm 0.066$ & $0.558 \pm 0.888$ \\
\hline 2-oxoglutarate & $2.426-2.467(\mathrm{t})$ & $1.440 \pm 0.619$ & $1.877 \pm 1.110$ \\
\hline 3-hydroxybutyrate & $2.298-2.306(q)$ & $0.083 \pm 0.036$ & $0.350 \pm 0.381$ \\
\hline \multicolumn{4}{|c|}{ ND fed diabetic rats versus HFD fed diabetic rats with metformin treatment } \\
\hline Compound & $\begin{array}{l}\text { Chemical shift, } \\
\text { ppm }\end{array}$ & $\begin{array}{l}\text { Relative Intensity } \\
\text { DNM }\end{array}$ & $\begin{array}{l}\text { Relative Intensity } \\
\text { DHM }\end{array}$ \\
\hline \multirow[t]{2}{*}{ Citrate } & $2.526-2.572(d)$ & $1.450 \pm 0.921$ & $3.097 \pm 1.582$ \\
\hline & $2.678-2.722(d)$ & $0.918 \pm 0.566$ & $1.783 \pm 0.882$ \\
\hline \multirow[t]{2}{*}{ Allantoin } & $5.388-5.409(s)$ & $0.276 \pm 0.058$ & $0.289 \pm 0.052$ \\
\hline & $6.007-6.054(s)$ & $0.709 \pm .216$ & $1.332 \pm 0.621$ \\
\hline Methylamine & $2.607-2.613(\mathrm{~s})$ & $0.120 \pm 0.048$ & $0.236 \pm 0.109$ \\
\hline Sarcosine & $2.723-2.728(\mathrm{~s})$ & $0.410 \pm 0.101$ & $0.789 \pm 0.283$ \\
\hline Methylguanidine & $2.825-2.848(s)$ & $0.289 \pm 0.058$ & $0.598 \pm 0.185$ \\
\hline Creatinine & $4.056-4.065(\mathrm{~s})$ & $1.065 \pm 0.197$ & $2.131 \pm 0.930$ \\
\hline \multirow[t]{2}{*}{ Lactate } & $1.322-1.348$ (d) & $15.165 \pm 14.421$ & $2.577 \pm 3.866$ \\
\hline & $4.100-4.149$ (q) & $7.713 \pm 7.082$ & $1.717 \pm 2.216$ \\
\hline
\end{tabular}

NN (control rat fed with ND); NH (control rat fed with HFD); DN (diabetic rat fed with ND); DNM (Diabetic rat fed with ND treated with metformin); DH (diabetic rat fed with HFD); DHM (diabetic rat fed with HFD treated with metformin). s, singlet; d, doublet; t, triplet; q, quartet.

citrate, cis-aconitate, 2-oxoglutarate, and succinate levels were identified in diabetic rats, which may imply the reduction of TCA cycle. On the other hand, metformin treatment on diabetic rats may reinstate the TCA cycle, where increased levels of citrate and succinate were detected in rats' urine. The effect of HFD on TCA cycle of diabetes rats was not certain; where an increase level of citrate and a decrease level of
Table 4. Metabolites that were altered by diabetes condition.

\begin{tabular}{|c|c|c|c|}
\hline \multicolumn{4}{|c|}{ Control rat fed on ND versus diabetic rat fed on ND } \\
\hline Compound & $\begin{array}{l}\text { Chemical shift, } \\
\text { ppm }\end{array}$ & $\begin{array}{l}\text { Relative Intensity } \\
\text { NN }\end{array}$ & $\begin{array}{l}\text { Relative Intensity } \\
\text { DN }\end{array}$ \\
\hline \multirow[t]{3}{*}{ Hippurate } & $7.536-7.564(\mathrm{t})$ & $3.045 \pm 1.867$ & $0.628 \pm 0.336$ \\
\hline & $7.622-7.665(t)$ & $1.533 \pm 0.919$ & $0.258 \pm 0.180$ \\
\hline & $7.823-7.86(d)$ & $3.402 \pm 2.004$ & $0.580 \pm 0.410$ \\
\hline \multirow[t]{2}{*}{ Citrate } & $2.529-2.568(d)$ & $7.066 \pm 4.984$ & $1.749 \pm 0.565$ \\
\hline & $2.66302 .713(d)$ & $6.875 \pm 4.495$ & $1.770 \pm 0.634$ \\
\hline Lactate & $1.327-1.346(d)$ & $1.475 \pm 2.126$ & $0.508 \pm 0.502$ \\
\hline \multirow[t]{2}{*}{ Allantoin } & $5.411-5.42(\mathrm{~s})$ & $3.150 \pm 0.955$ & $1.168 \pm 0.386$ \\
\hline & $6.016-6.052(\mathrm{~s})$ & $0.926 \pm 0.419$ & $0.264 \pm 0.140$ \\
\hline \multirow[t]{2}{*}{ Creatinine } & $3.037-3.052(\mathrm{~s})$ & $9.055 \pm 4.531$ & $0.767 \pm 0.417$ \\
\hline & $4.049-4.058(\mathrm{~s})$ & $4.387 \pm 2.379$ & $0.611 \pm 0.282$ \\
\hline Succinate & $2.403-2.414$ (s) & $1.530 \pm 0.689$ & $0.440 \pm 0.228$ \\
\hline Sarcosine & $2.723-2.731(\mathrm{~s})$ & $0.867 \pm 0.425$ & $0.176 \pm 0.066$ \\
\hline 2-oxoglutarate & $2.429-2.466(t)$ & $2.970 \pm 1.250$ & $1.452 \pm 0.650$ \\
\hline \multicolumn{4}{|c|}{ Control rat fed on HFD versus diabetic rat fed on HFD } \\
\hline Compound & $\begin{array}{l}\text { Chemical shift, } \\
\text { ppm }\end{array}$ & $\begin{array}{l}\text { Relative Intensity } \\
\mathrm{NH}\end{array}$ & $\begin{array}{l}\text { Relative Intensity } \\
\text { DH }\end{array}$ \\
\hline \multirow[t]{2}{*}{ Creatinine } & $3.036-3.049$ (s) & $8.244 \pm 4.993$ & $1.382 \pm 0.430$ \\
\hline & $4.046-4.058(\mathrm{~s})$ & $5.837 \pm 3.685$ & $1.043 \pm 0.271$ \\
\hline \multirow[t]{2}{*}{ Citrate } & $2.519-2.566(d)$ & $4.010 \pm 2.648$ & $2.157 \pm 0.983$ \\
\hline & $2.667-2.707(d)$ & $4.077 \pm 2.964$ & $2.004 \pm 0.842$ \\
\hline Allantoin & $5.385-5.404(\mathrm{~s})$ & $3.453 \pm 1.704$ & $1.553 \pm 0.350$ \\
\hline Phenylacetylglycine & $7.344-7.384(t)$ & $0.174 \pm 0.106$ & $0.052 \pm 0.018$ \\
\hline \multirow[t]{2}{*}{ Hippurate } & $7.535-7.578(t)$ & $2.478 \pm 1.633$ & $0.821 \pm 0.276$ \\
\hline & $7.824-7.851(d)$ & $2.645 \pm 1.796$ & $0.842 \pm 0.312$ \\
\hline Sarcosine & $2.721-2.728(\mathrm{~s})$ & $0.992 \pm 0.670$ & $0.316 \pm 0.094$ \\
\hline Cis-aconitate & $5.688-5.697(t)$ & $0.984 \pm 0.710$ & $0.390 \pm 0.114$ \\
\hline Succinate & $2.401-2.415(\mathrm{~s})$ & $2.672 \pm 2.503$ & $0.594 \pm 0.224$ \\
\hline Acetylglycine & $1.980-1.991(\mathrm{~s})$ & $0.593 \pm 0.382$ & $0.172 \pm 0.032$ \\
\hline 2-oxoglutarate & $2.427-2.464(t)$ & $2.523 \pm 1.819$ & $2.316 \pm 0.899$ \\
\hline Unknown & $1.198-1.203(\mathrm{~s})$ & $0.125 \pm 0.064$ & $1.964 \pm 4.670$ \\
\hline Unknown & $1.216-1.223(\mathrm{~s})$ & $0.163 \pm 0.103$ & $1.974 \pm 4.710$ \\
\hline Unknown & $2.034-2.044(\mathrm{~s})$ & $0.877 \pm 0.737$ & $0.304 \pm 0.112$ \\
\hline Unknown & $2.278-2.284(\mathrm{~s})$ & $0.224 \pm 0.141$ & $1.892 \pm 4.275$ \\
\hline
\end{tabular}

NN (control rat fed with ND); NH (control rat fed with HFD); DN (diabetic rat fed with ND).

s, singlet; d, doublet; t, triplet.

2-oxoglutarate were detected, this data may indicate that besides TCA cycle, the metabolites were the results of other pathways.

2-oxoglutarate (2-OG) or $\alpha$-ketoglutarate is the essential nitrogen transporter in the metabolic pathways. 2-OG is generated from isocitrate and decarboxylated into succinyl-CoA in TCA cycle. Urinary 2-OG level was reduced in diabetic rats and in diabetic rats fed with HFD. In contrary, 2-OG levels were increased in control rats fed with HFD. These contradicting effects of HFD on 2-OG levels in control rats and diabetic rats may be attributed to the diabetes condition itself that lowered the 2OG levels [34].

Urinary lactate was found lower in diabetic rats and diabetic rats fed with HFD, while metformin treatment increased urinary lactate levels. Diabetes reduces lactate production [35, 36]. Feeding of HFD may divert energy metabolism from glucose consumption to fatty acids consumption and caused a fall in urinary lactate by HFD. Metformin's role in improving glucose homeostasis in body may alter energy metabolism, in which we detected increased level of lactate in diabetic rats treated with metformin.

Fatty acid $\beta$-oxidation and ketogenesis may possibly enhance by HFD under diabetic condition. This was revealed by the increased levels of 
Table 5. Metabolites that were altered by metformin treatment on diabetes rats. DN (diabetic rat fed with ND).

Diabetic rats fed on ND with metformin treatment versus Diabetic rats fed on ND without metformin treatment

\begin{tabular}{llll}
\hline Compound & $\begin{array}{l}\text { Chemical shift, } \\
\text { ppm }\end{array}$ & $\begin{array}{l}\text { Relative Intensity } \\
\text { DN }\end{array}$ & $\begin{array}{l}\text { Relative Intensity } \\
\text { DNM }\end{array}$ \\
\hline Lactate & $1.323-1.344(\mathrm{~d})$ & $0.508 \pm 0.511$ & $29.786 \pm 28.886$ \\
\hline 3- & $4.100-4.1492(\mathrm{q})$ & $0.424 \pm 0.167$ & $8.503 \pm 7.712$ \\
hydroxybutyrate & $1.194-1.213(\mathrm{~d})$ & $0.049 \pm 0.014$ & $2.799 \pm 2.518$ \\
\hline Diabetic rats fed on HFD with metformin treatment versus Diabetic rats fed on HFD \\
without metformin treatment & & \\
\hline Compound & Chemical shift, & Relative Intensity & Relative Intensity \\
& ppm & DH & DHM \\
\hline Lactate & $1.319-1.348(\mathrm{~d})$ & $0.294 \pm 0.0 .99$ & $5.089 \pm 7.722$ \\
\hline Allantoin & $5.39-5.409(\mathrm{~s})$ & $1.507 \pm 0.470$ & $3.606 \pm 1.354$ \\
& $6.012-6.071(\mathrm{~s})$ & $0.389 \pm 0.141$ & $1.363 \pm 0.682$ \\
\hline 3- & $1.193-1.212(\mathrm{~d})$ & $7.639 \pm 13.981$ & $1.553 \pm 1.552$ \\
hydroxybutyrate & $2.317-2.405(\mathrm{q})$ & $2.571 \pm 4.336$ & $1.045 \pm 0.611$ \\
& $4.135-4.193(\mathrm{~s})$ & $1.518 \pm 2.557$ & $0.688 \pm 0.384$ \\
\hline Citrate & $2.528-2.586(\mathrm{~d})$ & $2.699 \pm 1.535$ & $4.924 \pm 2.428$ \\
\hline Succinate & $2.679-2.720(\mathrm{~d})$ & $2.538 \pm 1.497$ & $4.652 \pm 2.262$ \\
\hline Sarcosine & $2.407-2.413(\mathrm{~s})$ & $0.408 \pm 0.175$ & $0.899 \pm 0.484$ \\
\hline Creatinine & $2.722-2.726(\mathrm{~s})$ & $0.265 \pm 0.113$ & $0.804 \pm 0.320$ \\
\hline & $4.053-4.066(\mathrm{~s})$ & $0.877 \pm 0.371$ & $2.157 \pm 0.865$ \\
\hline
\end{tabular}

DN (diabetic rat fed with ND); DNM (Diabetic rat fed with ND treated with metformin); DH (diabetic rat fed with HFD); DHM (diabetic rat fed with HFD treated with metformin)

s, singlet; d, doublet; q, quartet.

ketone bodies (acetoacetate and acetone and 3-hydroxybutyarate) in diabetic rats fed with HFD. Under the condition of high fatty acid levels, ketogenesis will be a preferred pathway as TCA cycle capacity is overwhelmed [37]. Increased levels of ketone bodies can be an indication that HFD induced a greater stress on diabetes condition compared to ND. This is because these ketone bodies are induced under oxidative stress [38].

Taurine level was found decreased in control rats fed with HFD. Taurine is the major metabolite in bile acid metabolism. Taurine involves in cholesterol and bile salts conjugation, osmoregulation, antioxidation, ion movement, signalling modulation, membrane stabilisation, and neurotransmitters modulation [39]. Taurine is a by-product of the cysteine sulfonic acid pathway [40], that was suppressed in HFD induced obese mice [41].

Gut microbiome plays a significant role in human health as it is involved in various metabolic functions. Gut microbes produce biologically active metabolites through its energy harvesting and storage ability [42]. HFD and diabetes were associated with a change of gut bacterial composition, which can be recognised by specific metabolites [43, 44]. In this study, HFD fed control rats showed an increase in urinary phenylacetylglycine and a decrease in hippurate. In contrary, diabetic rats showed a decrease of phenylacetylglycine, acetylglycine and also hippurate. These results suggested that HFD may alter gut microbiome profiles in the rats. Hippurate level is associated with diet intake as it is a sensitive indicator for diet [45] and lower level of hippurate was reported in type 2 diabetes patients [46]. Acetylglycine is a product of pheylacetylglycine, while pheylacetylglycine is a biomarker for phospholipid accumulation [47], which is also associated with fat content in the body.

Choline is an important nutrient for cellular structure integrity, methyl metabolism and the transport/metabolism of lipid in the body $[48,49]$ and choline metabolism is strongly associated with gut microbiota activity. Trimethylamine-N-oxide (TMAO), methylamine and sarcosine are the products of choline metabolism [50]. In this study, urinary TMAO level was lower in control rats fed with HFD compared to those fed with ND, which may suggest the suppression of choline metabolism by HFD. Metformin treatment on HFD-fed diabetic rats showed an increase levels of methylamine and sarcosine, which indicate metformin treatment reverts choline metabolism by HFD.

Creatinine is the breakdown/waste product of creatine from muscle and protein metabolism. In this study, urinary creatinine level was lower in control rats fed with HFD compared to its counterpart on ND. It was also lower in diabetic rats compared with control rats. In contrary, creatinine levels was higher in both diabetic rats fed with HFD with/ without metformin treatment compared to those fed with ND, and in metformin treated diabetic rats fed with HFD compared to its counterpart without metformin treatment. HFD increases urinary creatinine excretion [51, 52, 53]. Increased level of creatinine indicates renal dysfunction [54], a condition when diabetes progressed and injured the kidney. This study was carried out at the early phase of diabetes, the low level of creatinine in diabetic rats indicated that kidney injury has not occurred at this early phase of the disease. Therefore, the increased level of creatinine observed in this study may solely due to the intake of HFD. Methylguanidine is formed due to the oxidation of creatinine [55, 56, 57]. In this study, we see a parallel level of methylguanidine and creatinine, where urinary level of methylguanidine was higher in diabetic rats fed with HFD treated with metformin than its counterpart fed on ND. Methylguanidine is a uremic toxin that accumulates in the body fluid of uremic patients or kidney failure patients [58].

Allantoin is generated from non-enzymatic oxidation of uric acid with ROS [59]. It is a biomarker of oxidative stress [60]. Plasma allantoin is higher in people with diseases related to oxidative stress such as rheumatoid arthritis, diabetes, and stroke [61, 62, 63, 64]. Urinary allantoin excretion also accurately reflects GFR as it is not reabsorbed in the proximal tubule of kidneys [65]. Therefore, oxidative stress and kidney function (GFR) will affect the allantoin level in urine. In this study, urinary allantoin level was higher in HFD diabetic rats with/without metformin treatment compared to their counterparts fed with ND, and higher in HFD-fed diabetic rat with metformin treatment compared to its counterpart without metformin treatment. In contrary, the level was lower in diabetic rats compared to control rats. HFD and diabetes increase ROS and oxidative stress $[66,67]$. The association of allantoin with HFD implies HFD may induce stress to the animals, both diabetic and healthy at the early phase of HFD consumption.

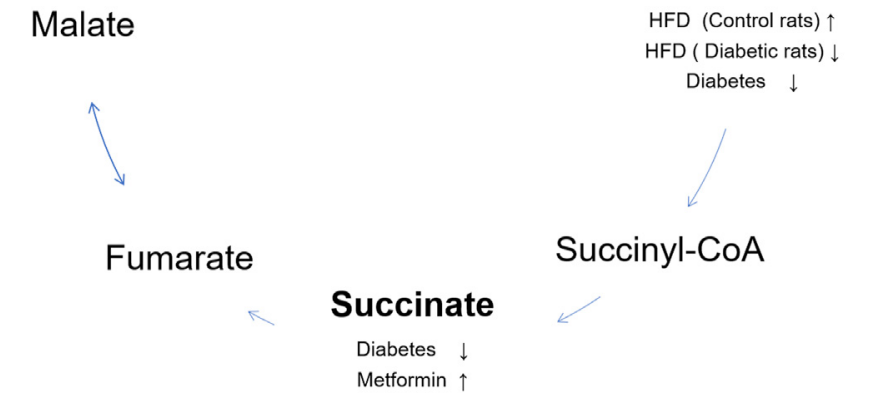

Figure 3. TCA cycle and its metabolites (adapted from Ryan et al. (2019) [32]). 


\section{Conclusion}

In this study, we aimed to investigate the impact of palm oil-enriched HFD consumption on diabetes condition at the early phase of the disease. The data obtained revealed that continuous consumption of HFD for a short period has adversely altered the metabolic pathways of the rats, both healthy and diabetic. The metabolites profiles of healthy and diabetic rats indicated that palm oil-enriched HFD induced oxidative stress to the animals. This effect was more prominent in the diabetes rats, where the increased excretion of creatinine and allontoin has marked the early kidney injury in diabetic rats. On the other hand, metformin treatment combined with normal diet lessened the physiological stress caused by diabetes disease. It is noteworthy that this current study is at preliminary stage, and the interpretation of the data is limited to the known metabolism pathways. Therefore, we acknowledge the possibility that the metabolites may be the products of other metabolism sources. Future study on the long-term effect of palm oil-enriched HFD on insulin sensitivity is worth investigating.

Declarations

Author contribution statement

Xuan-Yi Sim: Performed the experiments; Analyzed and interpreted the data; Wrote the paper.

Baharudin Ibrahim: Analyzed and interpreted the data; Contributed reagents, materials, analysis tools or data.

Lay-Harn Gam: Conceived and designed the experiments; Contributed reagents, materials, analysis tools or data; Wrote the paper.

Funding statement

This work was supported by Universiti Sains Malaysia (USM RUI topdown grant 1001/PFARMASI/870034).

Data availability statement

Data included in article/supplementary material/referenced in article.

Declaration of interests statement

The authors declare no conflict of interest.

Additional information

No additional information is available for this paper.

\section{Acknowledgements}

We would like to thank Dr. Peter Greimel from RIKEN Center for Brain Science, Wako, Japan for his advice and suggestion on the experimental design.

\section{References}

[1] P. Saeedi, I. Petersohn, P. Salpea, B. Malanda, S. Karuranga, N. Unwin, S. Colagiuri, L. Guariguata, A.A. Motala, K. Ogurtsova, J.E. Shaw, D. Bright, R. Williams, Global and regional diabetes prevalence estimates for 2019 and projections for 2030 and 2045: results from the International diabetes Federation diabetes atlas, 9th edition, Diabetes Res. Clin. Pract. 157 (2019).

[2] A.S. Al-Goblan, M.A. Al-Alfi, M.Z. Khan, Mechanism linking diabetes mellitus and obesity, Diabetes. Metab. Syndr. Obes. 7 (2014) 587-591.

[3] E.T. Kennedy, S.A. Bowman, R. Powell, Dietary-fat intake in the US population, J. Am. Coll. Nutr. 18 (1999) 207-212.

[4] R. Sambanthamurthi, K. Sundram, Y.-A. Tan, Chemistry and biochemistry of palm oil, Prog. Lipid Res. 39 (2000) 507-558.

[5] D.O. Edem, Palm oil: biochemical, physiological, nutritional, hematological and toxicological aspects: a review, Plant Foods Hum. Nutr. 57 (2002) 319-341.
[6] R.G. Jensen, Lipids in human milk, Lipids 34 (1999) 1243-1271.

[7] Y.S. Oh, G.D. Bae, D.J. Baek, E.-Y. Park, H.-S. Jun, Fatty acid-induced lipotoxicity in pancreatic beta-cells during development of type 2 diabetes, Front. Endocrinol. 9 (2018) 384.

[8] J.J. DiNicolantonio, J.H. O'Keefe, Good fats versus bad fats: a comparison of fatty acids in the promotion of insulin resistance, inflammation, and obesity, Mo. Med. 114 (2017) 303-307. https://pubmed.ncbi.nlm.nih.gov/30228616.

[9] P. Thomas, K.A. Leslie, H.J. Welters, N.G. Morgan, Long-chain saturated fatty acid species are not toxic to human pancreatic $\beta$-cells and may offer protection against pro-inflammatory cytokine induced $\beta$-cell death, Nutr. Metab. 18 (2021) 9.

[10] F. Magkos, M. Yannakoulia, J.L. Chan, C.S. Mantzoros, Management of the metabolic syndrome and type 2 diabetes through lifestyle modification, Annu. Rev. Nutr. 29 (2009) 223-256.

[11] R. Song, Mechanism of metformin: a tale of two sites, Diabetes Care 39 (2016) 187-189.

[12] C.J. Bailey, Metformin —an update, Gen. Pharmacol. Vasc. Syst. 24 (1993) 1299-1309.

[13] J.K. Nicholson, J.C. Lindon, E. Holmes, "Metabonomics": understanding the metabolic responses of living systems to pathophysiological stimuli via multivariate statistical analysis of biological NMR spectroscopic data, Xenobiotica 29 (1999) 1181-1189.

[14] A. Tebani, S. Bekri, Paving the way to precision nutrition through metabolomics, Front. Nutr. 6 (2019) 41.

[15] S. Bouatra, F. Aziat, R. Mandal, A.C. Guo, M.R. Wilson, C. Knox, T.C. Bjorndahl, R. Krishnamurthy, F. Saleem, P. Liu, Z.T. Dame, J. Poelzer, J. Huynh, F.S. Yallou, N. Psychogios, E. Dong, R. Bogumil, C. Roehring, D.S. Wishart, The human urine metabolome, Plos One 8 (2013), e73076.

[16] E. Pujos-Guillot, J. Hubert, J.-F. Martin, B. Lyan, M. Quintana, S. Claude, B. Chabanas, J.A. Rothwell, C. Bennetau-Pelissero, A. Scalbert, B. Comte, S. Hercberg, C. Morand, P. Galan, C. Manach, Mass Spectrometry-based metabolomics for the discovery of biomarkers of fruit and vegetable intake: citrus fruit as a case study, J. Proteome Res. 12 (2013) 1645-1659.

[17] H. Gibbons, B.A. McNulty, A.P. Nugent, J. Walton, A. Flynn, M.J. Gibney, L. Brennan, A metabolomics approach to the identification of biomarkers of sugarsweetened beverage intake, Am. J. Clin. Nutr. 101 (2015) 471-477.

[18] D.H. May, S.L. Navarro, I. Ruczinski, J. Hogan, Y. Ogata, Y. Schwarz, L. Levy, T. Holzman, M.W. McIntosh, J.W. Lampe, Metabolomic profiling of urine: response to a randomised, controlled feeding study of select fruits and vegetables, and application to an observational study, Br. J. Nutr. 110 (2013) 1760-1770.

[19] M. Beckmann, A.J. Lloyd, S. Haldar, G. Favé, C.J. Seal, K. Brandt, J.C. Mathers, J. Draper, Dietary exposure biomarker-lead discovery based on metabolomics analysis of urine samples, Proc. Nutr. Soc. 72 (2013) 352-361.

[20] S. Zhang, G.A.N. Gowda, V. Asiago, N. Shanaiah, C. Barbas, D. Raftery, Correlative and quantitative $1 \mathrm{H}$ NMR-based metabolomics reveals specific metabolic pathway disturbances in diabetic rats, Anal. Biochem. 383 (2008) 76-84.

[21] L. Zhao, H. Gao, F. Lian, X. Liu, Y. Zhao, D. Lin, 1H-NMR-based metabonomic analysis of metabolic profiling in diabetic nephropathy rats induced by streptozotocin, Am. J. Physiol. Physiol. 300 (2011) F947-F956.

[22] M. Guan, L. Xie, C. Diao, N. Wang, W. Hu, Y. Zheng, L. Jin, Z. Yan, H. Gao, Systemic perturbations of key metabolites in diabetic rats during the evolution of diabetes studied by urine metabonomics, PloS One 8 (2013), e60409.

[23] A.A.B. Sajak, A. Mediani, A. Ismail, F. Abas, Metabolite variation in lean and obese streptozotocin (STZ)-Induced diabetic rats via $1 \mathrm{H}$ NMR-based metabolomics approach, Appl. Biochem. Biotechnol. 182 (2017) 653-668.

[24] A. Mediani, F. Abas, M. Maulidiani, A. Khatib, C.P. Tan, I.S. Ismail, K. Shaari, A. Ismail, N.H. Lajis, Metabolic and biochemical changes in streptozotocin induced obese-diabetic rats treated with Phyllanthus niruri extract, J. Pharmaceut. Biomed. Anal. 128 (2016) 302-312.

[25] T. Koriyama, S. Wongso, K. Watanabe, H. Abe, Fatty acid compositions of oil species affect the 5 basic taste perceptions, J. Food Sci. 67 (2002) 868-873.

[26] P.E. Aba, I.U. Asuzu, Glycosylated haemoglobin values of alloxan-induced diabetic rats treated with graded doses of Cussonia arborea extract, J. Appl. Anim. Res. 46 (2018) 1478-1482.

[27] Z. Wang, Y. Yang, X. Xiang, Y. Zhu, J. Men, M. He, Estimation of the normal range of blood glucose in rats, Wei Sheng Yan Jiu= J. Hyg. Res. 39 (2010) 133-137.

[28] M. Bylesjö, M. Rantalainen, O. Cloarec, J.K. Nicholson, E. Holmes, J. Trygg, OPLS discriminant analysis: combining the strengths of PLS-DA and SIMCA classification, J. Chemom. A J. Chemom. Soc. 20 (2006) 341-351.

[29] M. Kinaan, H. Ding, C.R. Triggle, Metformin: an old drug for the treatment of diabetes but a new drug for the protection of the endothelium, Med. Princ. Pract. 24 (2015) 401-415.

[30] A.G. Liu, N.A. Ford, F.B. Hu, K.M. Zelman, D. Mozaffarian, P.M. Kris-Etherton, A healthy approach to dietary fats: understanding the science and taking action to reduce consumer confusion, Nutr. J. 16 (2017) 1-15.

[31] A.-H. Emwas, E. Saccenti, X. Gao, R.T. McKay, V.A.P.M. Dos Santos, R. Roy, D.S. Wishart, Recommended strategies for spectral processing and post-processing of 1D (1)H-NMR data of biofluids with a particular focus on urine, Metabolomics 14 (2018) 31.

[32] D.G. Ryan, M.P. Murphy, C. Frezza, H.A. Prag, E.T. Chouchani, L.A. O'Neill, E.L. Mills, Coupling Krebs cycle metabolites to signalling in immunity and cancer, Nat. Metab. 1 (2019) 16-33.

[33] L.J. Sweetlove, K.F.M. Beard, A. Nunes-Nesi, A.R. Fernie, R.G. Ratcliffe, Not just a circle: flux modes in the plant TCA cycle, Trends Plant Sci. 15 (2010) 462-470.

[34] L.F. Huergo, R. Dixon, The emergence of 2-oxoglutarate as a master regulator metabolite, Microbiol. Mol. Biol. Rev. 79 (2015) 419-435. 
[35] G.E. Duggan, D.S. Hittel, C.W. Sensen, A.M. Weljie, H.J. Vogel, J. Shearer, Metabolomic response to exercise training in lean and diet-induced obese mice, J. Appl. Physiol. 110 (2011) 1311-1318.

[36] L.-C. Zhao, X.-D. Zhang, S.-X. Liao, H.-C. Gao, H.-Y. Wang, D.-H. Lin, A metabonomic comparison of urinary changes in Zucker and GK rats, J. Biomed. Biotechnol. 2010 (2010).

[37] H.M. White, The role of TCA cycle anaplerosis in ketosis and fatty liver in periparturient dairy cows, Anim. Open Access J. from MDPI. 5 (2015) 793-802.

[38] S.K. Jain, K. Kannan, G. Lim, R. McVie, J.A. Bocchini, Hyperketonemia increases tumor necrosis factor- $\alpha$ secretion in cultured U937 monocytes and type 1 diabetic patients and is apparently mediated by oxidative stress and cAMP deficiency, Diabetes 51 (2002) 2287-2293.

[39] T. Ito, S.W. Schaffer, J. Azuma, The potential usefulness of taurine on diabetes mellitus and its complications, Amino Acids 42 (2012) 1529-1539.

[40] K. Sumizu, Oxidation of hypotaurine in rat liver, Biochim. Biophys. Acta 63 (1962) $210-212$.

[41] H.J. Schirra, C.G. Anderson, W.J. Wilson, L. Kerr, D.J. Craik, M.J. Waters, A.M. Lichanska, Altered metabolism of growth hormone receptor mutant mice: a combined NMR metabonomics and microarray study, PloS One 3 (2008), e2764 e2764.

[42] F. Bäckhed, Host responses to the human microbiome, Nutr. Rev. 70 (2012) S14-S17.

[43] V. Lazar, L.-M. Ditu, G.G. Pircalabioru, A. Picu, L. Petcu, N. Cucu, M.C. Chifiriuc, Gut microbiota, host organism, and diet trialogue in diabetes and obesity, Front. Nutr. 6 (2019) 21.

[44] M. Palau-Rodriguez, S. Tulipani, M. Isabel Queipo-Ortuño, M. Urpi-Sarda, F.J. Tinahones, C. Andres-Lacueva, Metabolomic insights into the intricate gut microbial-host interaction in the development of obesity and type 2 diabetes, Front. Microbiol. 6 (2015) 1151.

[45] S.S. Heinzmann, C.A. Merrifield, S. Rezzi, S. Kochhar, J.C. Lindon, E. Holmes, J.K. Nicholson, Stability and robustness of human metabolic phenotypes in response to sequential food challenges, J. Proteome Res. 11 (2012) 643-655.

[46] R.M. Salek, M.L. Maguire, E. Bentley, D. V Rubtsov, T. Hough, M. Cheeseman, D. Nunez, B.C. Sweatman, J.N. Haselden, R.D. Cox, S.C. Connor, J.L. Griffin, A metabolomic comparison of urinary changes in type 2 diabetes in mouse, rat, and human, Physiol. Genom. 29 (2007) 99. LP - 108, http://physiolgenomics.physiolo gy.org/content/29/2/99.abstract.

[47] A.W. Nicholls, J.K. Nicholson, J.N. Haselden, C.J. Waterfield, A metabonomic approach to the investigation of drug-induced phospholipidosis: an NMR spectroscopy and pattern recognition study, Biomarkers 5 (2000) 410-423.

[48] J.K. Blusztajn, Choline, a vital amine, Science (80-.) 281 (1998) 794-795.

[49] S.H. Zeisel, K. Da Costa, P.D. Franklin, E.A. Alexander, J.T. Lamont, N.F. Sheard, A. Beiser, Choline, an essential nutrient for humans, Faseb. J. 5 (1991) 2093-2098.

[50] A.M. Asatoor, M.L. Simeshoff, The origin of urinary dimethylamine, Biochim. Biophys. Acta Gen. Subj. 111 (1965) 384-392.

[51] G.A. Herrera, E.A. Turbat-Herrera, J. Teng, Mesangial homeostasis and pathobiology: their role in health and disease, in: Contrib. Nephrol., 2011, pp. 6-22.

[52] C. Gomez-Guerrero, P. Hernandez-Vargas, O. Lopez-Franco, G.O. M, J. Egido, Mesangial cells and glomerular inflammation: from the pathogenesis to novel therapeutic approaches, Curr. Drug Targets - Inflamm. Allergy 4 (2005) 341-351.
[53] J.-Y. Chen, D.-Y. Jian, C.-C. Lien, Y.-T. Lin, C.-H. Ting, L.-K. Chen, T.-C. Hsu, H.M. Huang, Y.-T. Wu, T.-T. Kuan, Y.-W. Chao, L.-Y. Wu, S.-W. Huang, C.-C. Juan, Adipocytes play an etiological role in the podocytopathy of high-fat diet-fed rats, J. Endocrinol. 231 (2016) 109-120.

[54] S.J. Sinkeler, A.J. Kwakernaak, S.J.L. Bakker, S. Shahinfar, E. Esmatjes, D. de Zeeuw, G. Navis, H.J. Lambers Heerspink, Creatinine excretion rate and mortality in Type 2 Diabetes and nephropathy, Diabetes Care 36 (2013) 1489. LP - 1494.

[55] K. Ienaga, K. Nakamura, M. Yamakawa, Y. Toyomaki, H. Matsuura, T. Yokozawa, H. Oura, K. Nakano, The use of 13 C-labelling to prove that creatinine is oxidized by mammals into creatol and 5-hydroxy-1-methylhydantoin, J. Chem. Soc. Chem. Commun. (1991) 509-510.

[56] S. Nagase, K. Aoyagi, M. Sakamoto, K. Takemura, T. Ishikawa, M. Narita, Biosynthesis of methylguanidine in the hepatic microsomal fraction, Nephron 62 (1992) 182-186.

[57] H. Ozasa, S. Horikawa, K. Ota, Methylguanidine synthase from rat kidney is identical to long-chain L-2-hydroxy acid oxidase, Nephron 68 (1994) 279.

[58] F. Wang, B. Yang, G.H. Ling, C. Yao, Y.S. Jiang, Methylguanidine cytotoxicity on HK-2 cells and protective effect of antioxidants against MG-induced apoptosis in renal proximal tubular cells in vitro, Ren. Fail. 32 (2010) 978-985.

[59] H. Kaur, B. Halliwell, Action of biologically-relevant oxidizing species upon uric acid. Identification of uric acid oxidation products, Chem. Biol. Interact. 73 (1990) 235-247.

[60] I.F.F. Benzie, W. Chung, B. Tomlinson, Simultaneous measurement of allantoin and urate in plasma: analytical evaluation and potential clinical application in oxidant: antioxidant balance studies, Clin. Chem. 45 (1999) 901-904.

[61] R. Kand'ár, P. Žáková, V. Mužáková, Monitoring of antioxidant properties of uric acid in humans for a consideration measuring of levels of allantoin in plasma by liquid chromatography, Clin. Chim. Acta 365 (2006) 249-256.

[62] R.C.S. Seet, C.-Y.J. Lee, B.P.L. Chan, V.K. Sharma, H.-L. Teoh, N. Venketasubramanian, E.C.H. Lim, W.-L. Chong, W.-F. Looi, S.-H. Huang, Oxidative damage in ischemic stroke revealed using multiple biomarkers, Stroke 42 (2011) 2326-2329.

[63] S. Yardim-Akaydin, A. Sepici, Y. Özkan, B. Simșek, V. Sepici, Evaluation of allantoin levels as a new marker of oxidative stress in Behcet's disease, Scand. J. Rheumatol. 35 (2006) 61-64.

[64] W.-Y. Chung, I.F.F. Benzie, Plasma allantoin measurement by isocratic liquid chromatography with tandem mass spectrometry: method evaluation and application in oxidative stress biomonitoring, Clin. Chim. Acta 424 (2013) $237-244$.

[65] J.P. Briggs, M.F. Levitt, R.G. Abramson, Renal excretion of allantoin in rats: a micropuncture and clearance study, Am. J. Physiol. Physiol. 233 (1977) F373-F381.

[66] N. Matsuzawa-Nagata, T. Takamura, H. Ando, S. Nakamura, S. Kurita, H. Misu, T. Ota, M. Yokoyama, M. Honda, K. Miyamoto, Increased oxidative stress precedes the onset of high-fat diet-induced insulin resistance and obesity, Metabolism 57 (2008) 1071-1077.

[67] B.L. Tan, M.E. Norhaizan, Effect of high-fat diets on oxidative stress, cellular inflammatory response and cognitive function, Nutrients 11 (2019) 2579. 